Supporting Information of

\title{
Two-Dimensional Carbon-based Auxetic Materials for Broad-Spectrum Metal-Ion Battery Anodes
}

Shuaiwei Wang a, Yubing Si a , Baocheng Yang a, Eli Ruckenstein ${ }^{\text {b }}$, Houyang Chen ${ }^{\text {b* }}$

${ }^{a}$ Henan Key Laboratory of Nanocomposites and Applications, Institute of Nanostructured Functional

Materials, Huanghe Science and Technology College, Zhengzhou 450006, China

${ }^{b}$ Department of Chemical and Biological Engineering, State University of New York at Buffalo,

Buffalo, New York 14260-4200, USA

* To whom correspondence should be addressed. Email: hchen23@buffalo.edu 


\section{Section S1. Computational methods}

Our density functional theory calculations were implemented using the Vienna $a b$ initio Simulation Package (VASP) ${ }^{1-2}$. Electron wave functions were described by projector augmented-wave $(\mathrm{PAW})^{3}$ pseudopotentials with a kinetic energy cutoff of $500 \mathrm{eV}$. The Perdew-Burke-Ernzerhof (PBE) functional with the Generalized Gradient Approximation (GGA) ${ }^{4}$ was employed to treat the electron exchangecorrelation interactions and the Heyd-Scuseria-Ernzerhof $(\text { HSE06) })^{5}$ functional is used to acquire more accurate electronic structures. $11 \times 7 \times 1 \mathrm{k}$-grid meshes were sampled in the Brillouin zone for structural relaxation, $21 \times 21 \times 1 \mathrm{k}$-grid meshes were adopted for electronic properties calculations, and $5 \times 5 \times 1 \mathrm{k}$-grid meshes were used for $\mathrm{Li} / \mathrm{Na} / \mathrm{K} / \mathrm{Ca}$-content dependent adsorption energies and open-circuit voltage calculations.

A vacuum space of $20 \AA$ is adopted to avoid interaction between neighboring layers. The convergence threshold for the force on each atom is $0.01 \mathrm{eV} / \AA$. The finite displacement method implemented in the Phonopy package ${ }^{6}$ and the climbing-image nudged elastic band method ${ }^{7-8}$ were employed to compute the Phonon data and to study the diffusion barriers of metal ions on the surface, respectively. 


\section{References}

(1) Kresse, G.; Furthmüller, J. Efficiency of Ab-initio Total Energy Calculations for Metals and Semiconductors using a Plane-wave Basis Set. Computat. Mater. Sci. 1996, $6(1), 15-50$.

(2) Kresse, G.; Furthmüller, J. Efficient Iterative Schemes for Ab initio Total-energy Calculations using a Plane-wave Basis Set. Phys.rev. B 1996, 54 (16), 11169.

(3) Kresse, G.; Joubert, D. From Ultrasoft Pseudopotentials to the Projector Augmented-Wave Method. Phys. Rev. B 1999, 59 (3), 1758.

(4) Wang, Y.; Perdew, J. P. Correlation Hole of the Spin-Polarized Electron Gas, with Exact Small-Wave-Vector and High-Density Scaling. Phys. Rev. B 1991, 44 (24), 13298.

(5) Heyd, J.; Scuseria, G. E.; Ernzerhof, M. Hybrid Functionals based on a Screened Coulomb Potential. J. Chem. Phys. 2003, 118 (18), 8207-8215.

(6) Togo, A.; Tanaka, I. First Principles Phonon Calculations in Materials Science. Scripta Materialia 2015, 108, 1-5.

(7) Henkelman, G.; Uberuaga, B. P.; Jónsson, H. A Climbing Image Nudged Elastic Band Method for Finding Saddle Points and Minimum Energy Paths. J. Chem. Phys. 2000, 113 (22), 9901-9904.

(8) Henkelman, G.; Jónsson, H. Improved Tangent Estimate in the Nudged Elastic Band Method for Finding Minimum Energy Paths and Saddle points. J. Chem. Phys. 2000, 113 (22), 9978-9985. 